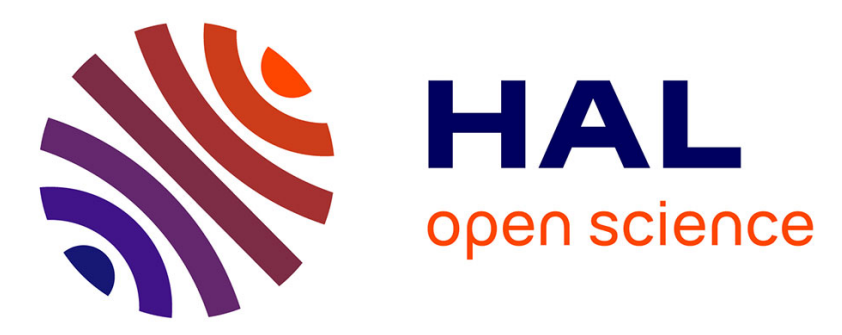

\title{
Genetic diversity and its value: conservation genetics meets economics
}

\author{
Noël Bonneuil, Raouf Boucekkine
}

\section{To cite this version:}

Noël Bonneuil, Raouf Boucekkine. Genetic diversity and its value: conservation genetics meets economics. Conservation Genetics Resources, 2020, 12 (1), pp.141-151. 10.1007/s12686-019-01113-y . hal-02417600

\section{HAL Id: hal-02417600 \\ https://hal-amu.archives-ouvertes.fr/hal-02417600}

Submitted on 30 Apr 2020

HAL is a multi-disciplinary open access archive for the deposit and dissemination of scientific research documents, whether they are published or not. The documents may come from teaching and research institutions in France or abroad, or from public or private research centers.
L'archive ouverte pluridisciplinaire HAL, est destinée au dépôt et à la diffusion de documents scientifiques de niveau recherche, publiés ou non, émanant des établissements d'enseignement et de recherche français ou étrangers, des laboratoires publics ou privés. 


\title{
Genetic diversity and its value: conservation genetics meets economics
}

\author{
Noël Bonneuil ${ }^{1} \cdot$ Raouf Boucekkine ${ }^{2,3}$
}

\begin{abstract}
Does drawing economic benefit from nature impinge on conservation? This has been a subject of controversy in the litera-ture. The article presents a management method to overcome this possible dilemma, and reconcile conservation biology with economics. It is based on recent advances in the mathematical theory of dynamic systems under viability constraints. In the case of a one-locus two-allele plant coexisting with a one-locus two-allele parasite, the method provides a rule for deciding when and to what extent the resistant or the susceptible strain should be cultivated, in the uncertain time-varying presence of the parasite. This is useful for preventing the fixation of the susceptible allele-and thereby limiting the plant's vulnerability in the medium term, should the parasite reappear. The method thus provides an aid to decision for economic and ecology-friendly profitability.
\end{abstract}

Keywords $C$-viability $\cdot$ Sustainability $\cdot$ Genetic diversity $\cdot$ Genetic distance $\cdot$ Genetic resistance

Mathematics Subject Classification 93C83 $93 \mathrm{C} 95 \cdot 92 \mathrm{D} 25 \cdot 92 \mathrm{D} 10$

JEL Classification Q01 · Q20 · Q56

\section{Introduction}

Preserving genetic diversity corresponds to maintaining the proportions of alleles within a certain range. An allele is a variant expression of a gene, which itself is a locus of DNA. Different alleles of a same gene may have different properties, notably in terms of susceptibility or resistance to parasites.

Calls to "halt biodiversity loss" and "conserve and sustainably use the oceans, seas and marine resources" are now on the United Nations' "Goals for Sustainable Development" agenda. Sustaining genetic diversity raises the question of genetic diversity's economic value, a question made intricate

Noël Bonneuil

bonneuil@ined.fr

1 Institut national d'études démographiques, and École des hautes études en sciences sociales, Paris, France

2 Cnrs, Éhess, Centrale Marseille, Amse, and Iméra, Aix-Marseille University, Marseille, France

3 Institut universitaire de France, Paris, France by the presence of both quantitative and qualitative facets (Ten Kate and Laird 2004). The economic literature on biodiversity and the related measurement methods are abundant (Nijkamp et al. 2008). They may be controversial: for example, Spash (2015) denounces the use of economics as a way to legitimize destruction under the cover of rationalizing nature conservation by building trade-offs which ultimately favor developers to the detriment of conservation. Conversely, Meinarda et al. (2016) argue that economics are diverse and that monetary valuation would be beneficial to conservation biologists. Edwards and Abivardi (1998) include pest control in ecosystem services, and argue that economic analysis can contribute to conservation goals and to the preservation of biological and genetic diversity.

We contribute to the debate by presenting a method of managing genetic diversity that gives priority to preservation while taking advantage of it economically. We describe how an optimal economic return can be obtained while preserving allelic polymorphism in a typical one-locus two-allele plant coexisting with a one-locus two-allele parasite. The mathematics of maintaining a differential system within a closed set was pioneered by Perron (1915) and Nagumo 
(1942). These authors characterized the directions a system must take so as to remain within a given set. The topic was developed in biological contexts by Bonneuil (1998, 2003, 2012a) and Bonneuil and Saint-Pierre (2000, 2002), and in economics by Bonneuil (1994a, b, 2010) and Bonneuil and Boucekkine $(2014,2016)$.

We build on Bonneuil (2012b), who reconciled optimality and maintenance within a given set, so as to define and compute the set of " $C$-viable" optima, which are optima for an inter-temporal criterion attained by a trajectory remaining within a given set, here identified as polymorphism. In doing so, we will translate what this key property means in terms of genetic distance. We no longer situate the question of genetic diversity combined with economic rationality at equilibria, but in transient time, which is the time most suited to describing restless systems such as biological ones.

Theoretical biology has indeed much focused on equilibria, reducing the problem of genetic diversity to the question of the ultimate preservation of alleles under the joint action of migration and selection (references in Bonneuil 2012a). Here, we argue that the economic value defining genetic diversity is the maximal inter-temporal benefit obtained by harvesting each allelic form of the plant, while remaining in a given range over time, and this while fitness values vary unpredictably, due to the weather, stress, fluctuations of the environment, and genetic heterogeneity (Pianka 1978).

Concerning the economics of genetics, in the "Noah's ark problem," species are valued both by their genetic distances and by their utility, reflecting "aesthetic and existence values" (Weitzman 1998). Brock and Xepapadeas (2003) developed on Heal (2000), who considered genetic diversity as a commodity: a species is "desirable" for the services it can render to humankind. Instead of defining the value of genetic diversity by a function based on pairwise distances between species' DNA (Weitzman 1992, 1993; Solow et al. 1993), which is a technique borrowed from geneticists (e.g. Slatkin and Hudson 1991), they computed the value of genetic diversity as the Bellman value function of optimal resource management at the steady state. This function gives the cumulated payoff to be expected at the time horizon and resulting from the initial conditions and from the decision sequence made from now until the time horizon. The optimal value function is obtained with the optimal decision sequence, and then depends only on the initial conditions. We give an explicit formula in Eq. (1). In their model of a one-locus diallelic two-species case, Brock and Xepapadeas (2003) postulated that the mortality rate of each plant is a function of the proportion of one of the two alleles. They assumed random mating and fixed survival probabilities. To maximize an intertemporal economic criterion under biological dynamics, they situated themselves at the optimal steady state.

However, as Law and Morton (1996, p. 763) pointed out, "it is not clear that local asymptotic stability is an appropriate condition for coexistence, for species may coexist without tending to an equilibrium point" (Williamson 1987; Hastings 1988); and the "dynamics close to an interior equilibrium point are not the main issue for coexistence." Fitness values vary, and polymorphism may not converge. Moreover, observing a state $x_{0}$, whose coordinates are the frequencies of the various alleles, there is no reason that $x_{0}$ should correspond to a steady state. The economic value then corresponds not to $x_{0}$, but, at best, to the steady state which could be attained starting from $x_{0}$ and assuming that fitness values are constant. This is the analytical gap we wish to fill here: extend Brock and Xepapadeas's (2003) project of associating the value function, but with each possible state $x_{0}$ and by allowing fitness values to vary over time. We innovate by considering out-of-equilibrium dynamics, which are realistic when dealing with population genetics. To do this, we use the adequate mathematics of dynamic systems under $C$-viability constraints (Aubin 1991). ${ }^{1}$

We refrain from postulating ad hoc mechanisms, of which we have no knowledge (for as Morin and Lawler 1995 , p. 511 put it, "community ecology has a long tradition of conjectures"). We do not fix mortality rates, but take the possible fluctuations of these rates as participating in the uncertainty inherent in the variation of fitness values, for which we specify only their membership of some closed set. Brock and Xepapadeas (2003) also ignored the state constraints of having sufficient density above which the plant is to perpetuate itself and within which the allele distribution is "sufficiently" diverse. We shall specify state constraints, to be included in the maximization program.

We use the concept of " $C$-viability kernel" and " $C$-capture-viability kernel" to revisit the economic value of genetic diversity. The $C$-viability kernel is the set of all initial states from which there exists at least one trajectory remaining in the set of state constraints up to the time horizon (possibly infinite). The $C$-capture-viability kernel is the set of all initial states from which there exists at least one trajectory that remains in the set of state constraints up to the time horizon and attains a given set at that time. Alleles no longer have to be at the steady state, but instead their frequencies can vary under time-varying fitness values. So we are no longer in the context of the convergence of the (genetic) system towards a steady state, although the $C$-viability kernel may include steady states, but we focus on the process in transient time. To say it again, the sustainability of a system that varies over time amounts to identifying its $C$-viability kernel, which is

\footnotetext{
1 The term "viability analysis" has been used to address probabilistic estimates of population survival or extinction (Beissinger and McCullough 2002; Morris and Doak 2002; Wengera et al. 2017). Here, we refer to the term used in control theory (Aubin 1991). To avoid confusion, we use the term " $C$-viability" (as in Bonneuil and Saint-Pierre 2000, 2002).
} 
the set of states from which there is a chance of perpetuating the system, whereas a steady state may not be polymorphic as desired and a system may fluctuate while however preserving genetic diversity.

We present the genetic dynamic for the plant and its parasite, the equilibria, the basics of $C$-viability theory and the theoretical procedure yielding the viable maxima. We show the role played by the parameters and how viability constraints modify the interplay between the various associations between the parasite and plant alleles.

We build the dynamic set comprising all initial conditions from which there exists at least one optimal trajectory preserving genetic diversity. This set, called " $C$-captureviability kernel," provides regulation rules stipulating how the resistant and the susceptible strains must, at each time, be either spared or harvested. We then provide the harvest rates to apply as determined by this set, making them endogenous (rather than postulating some ad hoc endogeneity and then testing its capacity to produce genetic diversity and economic optimality). We establish how this set varies with the determinants (such as carrying capacity, population size, and costs of fitness and of being diseased), leading us then to characterize the effects of these covariates on genetic diversity and economic value in a general time-varying setting. A genetic distance, although it hides the influence of each component of the value function, has been used to measure diversity (Brock and Xepapadeas 2003). We show that, for a low prevalence of the infective parasite, the value function is higher when the genetic distance is small. When the infective parasite is sufficiently abundant, the value function is higher when the distance is high, which corresponds to equal proportions of the resistant and susceptible alleles. The distance may be used to set the initial conditions of the genetic diversity of the plant, considering the initial prevalence of the parasite.

\section{Model}

\section{Intertemporal maximal benefit under genetic dynamic}

We borrow from Moreno-Gàmez et al. (2013) for the genetic dynamic. Host and parasite are haploid with discrete generations. The host has two alleles, resistance (allele "RES"), in frequency $R_{t}$ at discrete-time generation $t$, and susceptibility (allele "res"), in frequency $r_{t}$. The parasite has two alleles, infectivity (allele "INF") in frequency $a_{t}$ and non-infectivity (allele "ninf") in frequency $A_{t}$.

As in Nuismer and Otto (2005) or Tellier and Brown (2011), each generation of the host encounters the parasite at random with probability $\rho$. Infective parasites harbor a cost of virulence $b$. Leonard (1977) reports several consistent
Table 1 Fitness values of hosts and parasites for the gene-for-gene model in a single population. Reproduced from Moreno-Gàmez et al. (2013)

\begin{tabular}{llllll}
\hline $\begin{array}{lllll}\text { Disease } \\
\text { prevalence }\end{array}$ & \multicolumn{2}{l}{ Genotype frequency } & & Fitness \\
\cline { 2 - 3 } \cline { 5 - 6 } & Host & Parasite & & Host & Parasite \\
\hline$\rho$ & $\operatorname{RES}\left(R_{t}\right)$ & $\operatorname{ninf}\left(A_{t}\right)$ & & $1-c_{f}$ & $1-c$ \\
& & $\operatorname{INF}\left(a_{t}\right)$ & & $\left(1-c_{f}\right)(1-s)$ & $1-b$ \\
& $\operatorname{res}\left(r_{t}\right)$ & $\operatorname{ninf}\left(A_{t}\right)$ & & $1-s$ & 1 \\
& & $\operatorname{INF}\left(a_{t}\right)$ & $1-s$ & $1-b$ \\
$1-\rho$ & $\operatorname{RES}\left(R_{t}\right)$ & & $1-c_{f}^{*}$ & n.a. \\
& $\operatorname{res}\left(r_{t}\right)$ & & 1 & n.a. \\
\hline
\end{tabular}

$\rho$ Disease prevalence; alleles for host resistance (RES) and susceptibility (res); alleles for pathogen non-infectivity (ninf) and infectivity (INF), $b$ cost of virulence, $c$ cost of inability to infect the allele RES of the host, $c_{f}$ cost of fitness when a resistance reaction is triggered, $c_{f}^{*}$ cost of fitness to resistant hosts that do not encounter a pathogen, $s$ cost of being diseased, $t$ generation n.a. not applicable

case studies where this cost is estimated. Non-infective parasites do not bear this cost, but if they encounter a resistant host they have a cost $c$ of not being able to infect it. It corresponds to the plant's successful resistance to the parasite and therefore to the reduction by a factor $c$ of the reproduction of the infective allele of the parasite (Leonard 1977; Tellier and Brown 2011). Leonard (1977) estimates this cost $c$ to be very close to 1 , which is the value we take, as Tellier and Brown (2007) did. For the host, being diseased has a cost $s$, equal to the reduction in the plant's reproductive fitness when being diseased. This occurs when the host has the susceptibility allele (res) and the parasite has the virulence allele (INF) (Tellier and Brown 2011).

Resistant hosts encountering parasites trigger a resistance reaction that incurs a cost of fitness $c_{f}$, irrespective of whether the defense is successful or not. Burdon and Thrall (2003) report a controversy as to whether fitness costs of resistance are necessary for the maintenance of resistance and virulence gene polymorphisms. Most models of genes include fitness costs associated with host resistance and pathogenic virulence genes. As the genetic dynamics are continuous in all parameters, the case $c_{f}=0$ is the limit case of small $c_{f}$. When hosts do not encounter the parasite, which happens with probability $1-\rho$, resistant hosts have a cost $c_{f}^{*}$. This corresponds to the basal cost of harboring the allele for resistance. Thus, $c_{f}=c_{f}^{*}+\epsilon$, where $\epsilon \geq 0$ is the cost of activating and expressing the defense genes, which are triggered by plant cells when they recognize pathogens during attacks by a pathogen $(\mathrm{Gu}$ et al. 2002). Parasites that do not encounter hosts do not survive, regardless of their genotypes. The fitness values of hosts and parasites are summarized in Table 1. All the costs and the encounter rate are assumed to be constant over time. The effect 
of the resistance of the host is to reduce the fitness of the noninfective parasite, passing from 1 to $1-c$, but at a cost $u$.

In the case where there are several generations of parasites per host generation, if there is pure allo-infection (the parasite was not born on the plant it infects), the behavior of the model is the same as for one generation (Tellier et al. 2014). Allo-infection involves many plant pathogens (Barrett 1980) and the single-generation model is easier to study. Postulating several generations of the parasite per host generation requires rethinking the model. The single-generation model we present is a reference, where the search for stability as in Tellier et al. (2014) or for maintenance in a given set defining genetic diversity as we do here is not confused by a complicated effect such as several generations of parasites per host generation.

The program for maximizing the economic benefit while preserving genetic diversity at time horizon $T$ is:

$\max _{h_{t}^{R}, h_{t}^{r}} \sum_{t=0}^{T} e^{-\xi t}\left(h_{t}^{R} R_{t}+h_{t}^{r} r_{t}\right)$,

where $\xi \geq 0$ is a discount rate used to reflect the preference for the present (a harvest today is worth more than a harvest tomorrow Henderson 2008), $h_{t}^{R}$ the harvest rate of the resistant allelic form RES at frequency $R_{t}$ at time $t$, and $h_{t}^{r}$ the harvest rate of the susceptible allelic form res at frequency $r_{t}$. The state variables are governed by the dynamics

$$
\left\{\begin{aligned}
R_{t+1}= & R_{t}\left(K_{R r}-R_{t}-r_{t}\right)\left(1+v^{H}\right)\left(1-h_{t}^{R}\right) \\
& \times\left(\rho\left(1-c_{f}\right)\left(A_{t}+(1-s) a_{t}\right)\right. \\
& \left.+(1-\rho)\left(1-c_{f}+\epsilon\right)\right)=: f_{1}\left(x_{t}, u\right) \\
r_{t+1}= & r_{t}\left(K_{R r}-R_{t}-r_{t}\right)\left(1+v^{H}\right)\left(1-h_{t}^{r}\right) \\
& \times\left(\rho(1-s)\left(A_{t}+a_{t}\right)+1-\rho\right)=: f_{2}\left(x_{t}, u\right) \\
A_{t+1}= & A_{t} \rho\left((1-c) R_{t}+r_{t}\right)\left(1+v_{t}^{P}\right)=: f_{3}\left(x_{t}, u\right) \\
a_{t+1}= & a_{t} \rho(1-b)\left(1+v_{t}^{P}\right)\left(R_{t}+r_{t}\right)=: f_{4}\left(x_{t}, u\right),
\end{aligned}\right.
$$

where, at time $t, K_{R r}$ is the carrying capacity, $v^{H}$ the fixed growth rate of the host, and $v_{t}^{P}$ the growth rate of the parasite, taken as varying unpredictably within a closed set $\left[v_{\min }^{P}, v_{\max }^{P}\right]$, so that the control variables are:

$h_{t}^{R} \in[0,1], \quad h_{t}^{r} \in[0,1]$,

and the perturbation is:

$v_{t}^{P} \in\left[v_{\min }^{P}, v_{\max }^{P}\right]$.

The state is $x(t):=\left(R_{t}, r_{t}, A_{t}, a_{t}\right)$, the control variable $u(t):=\left(h_{t}^{R}, h_{t}^{r}\right)$, and the perturbation $v(t):=v_{t}^{P}$. The state constraints are:
$\left\{\begin{array}{l}R_{t} \geq R_{\min }, \\ r_{t} \geq r_{\min }, \\ p_{\min } \leq \frac{R_{t}}{r_{t}} \leq p_{\max }\end{array}\right.$

where $p_{\min }$ and $p_{\max }$ are threshold values defining genetic diversity, $R_{\min }$ and $r_{\min }$ thresholds defining minimal abundance under which the allele RES or the allele res is considered to be rare. Our definition of genetic diversity by a mere interval of variation for $\frac{R_{t}}{r_{t}}$ is simply a translation of "not too little," "not too much" of each allele. It avoids more drastic constraints (such as $\frac{R_{t}}{r_{t}}=1$ ), which are inadequate in a timevarying environment.

Equation (2) is of the Lotka-Volterra type, because it includes a carrying capacity and compounds of hosts encountering parasites. However, it is complicated by the relationship of allele dominance within loci, by virulence or defense costs, and by the fact that encounters with the parasite have different effects, depending on the resistance or not and of infectivity or not. It is a development of the model used by Moreno-Gàmez et al. (2013), with the difference that we consider the population sizes of each allele instead of the mere proportions $R_{t} / r_{t}$ and $A_{t} / a_{t}$. Indeed, preserving genetic diversity requires that each allele is abundant enough. The proportion $R_{t} / r_{t}$ results from the dynamics of $R_{t}$ and $r_{t}$. This allows us to identify "sufficient" abundance of $R_{t}$ and $r_{t}$ but also "sufficient" levels of $R_{t} / r_{t}$ or $r_{t} / R_{t}$ as a set of constraints within which the system is to remain, under the control of harvest rates and under the attacks of the parasite, whose population grows unpredictably (between $v_{\text {min }}^{P}$ and $\left.v_{\max }^{P}\right)$. This yields our profound difference with classical Lotka-Volterra models: rather than searching for asymptotic patterns (including equilibria or limit cycles as it is commonly done), we focus on maintaining the system in a given set and in transient time, which may be appropriate to biology rather than a long or an infinite time, which is justified in physics where processes may converge quickly.

The maximization in (1) under the dynamics (2), control constraints $\{3,4\}$, and state constraints (5), is done by computing the upper boundary of the capture-viability kernel of an augmented dynamic, where the added state $y(t)$ starts from the value function (Bonneuil 2012b) [Eq. (15)].

With this system, we no longer need to define genetic diversity by reference to equilibrium, but we do it from any state. By using set-valued analysis resulting from (3) and (4), we take full consideration of the uncertainty inherent in the temporal variation of the populations (for example, we no longer need to postulate ad hoc mortality). Our model combines dynamic optimization and constraints, that is, economic benefit and genetic diversity. 


\section{Equilibrium}

If the control variables $h^{R}, h^{r}$, and the perturbation $v^{P}$ are constant, then System (2) has the single equilibrium

$\left\{\begin{array}{l}R^{*}=\frac{b}{\rho c(1-b)\left(1+\nu^{P}\right)}, \\ r^{*}=\frac{c-b}{\rho c(1-b)\left(1+\nu^{P}\right)}, \\ A^{*}=\frac{(\alpha-1) \xi_{2}+\xi_{1}}{\alpha}, \\ a^{*}=\frac{\xi_{2}-\xi_{1}^{\alpha}}{\alpha},\end{array}\right.$

where

$$
\begin{aligned}
\xi_{1}= & \frac{1}{\rho\left(1-c_{f}\right)}\left(\left(\left(K_{R r}-R^{*}-r^{*}\right)\left(1+v^{H}\right)\left(1-h^{R}\right)\right)^{-1}\right. \\
& \left.-(1-\rho)\left(1-c_{f}+\epsilon\right)\right), \\
\xi_{2}= & \frac{1}{\rho(1-s)}\left(\left(\left(K_{R r}-R^{*}-r^{*}\right)\left(1+v^{H}\right)\left(1 h^{R}\right)\right)^{-1}\right. \\
& -(1-\rho)) .
\end{aligned}
$$

This equilibrium may be located outside the set of constraints of (5), for example if $\frac{R^{*}}{r^{*}}=\frac{b}{c-b} \notin\left[p_{\min }, p_{\max }\right]$. In this case, Brock and Xepapadeas (2003) may affect a genetic diversity measure to the system, but computing the genetic diversity value for an equilibrium which is outside the set of constraints is contradictory. Besides, restricting genetic diversity as well as economic benefit to steady states is overly restrictive.

\section{C-viability theory: basic concepts}

The problem $\{(2),(3,(5))\}$ is equivalent to the recursive inclusion:

$$
\left\{\begin{array}{l}
x(t+1) \in F(x(t)) \\
\text { and } \\
\forall t=0, \ldots, T, x(t) \in K,
\end{array}\right.
$$

where

$$
\begin{aligned}
K & =\left\{x=(R, r, A, a) \in \mathbb{R}^{4+*} \mid p_{\min } \leq \frac{R}{r} \leq p_{\max } \text { and } R\right. \\
& \left.\geq R_{\min } \text { and } r \geq r_{\min }\right\}
\end{aligned}
$$

and

$$
\begin{gathered}
F(x)=\left\{\left(f_{1}(x, u, v), f_{2}(x, u, v), f_{3}(x, u, v), f_{4}(x, u, v)\right) \mid\right. \\
\left.u \in[0,1]^{2}, v \in\left[v_{\min }^{P}, v_{\max }^{P}\right]\right\},
\end{gathered}
$$

$$
\left(u(t), v(t)=\left(h_{t}^{R}, h_{t}^{r}, v_{t}^{P}\right) \in[0,1]^{2} \times\left[v_{\min }^{P}, v_{\max }^{P}\right] .\right.
$$

A state $x_{0}$ is said to be viable in $K$ under $F$ if there exists at least one solution $x($.$) of (8), starting from x(0)=x_{0}$ and remaining in $K$ up to the time horizon $T$. A set of $C$-viable states is called a $C$-viability domain; and there exists a maximal $C$-viability domain that includes all others (Aubin 1991). This set is the viability kernel $\operatorname{Viab}_{F}(K)$ (which is then a set of initial conditions):

$$
\begin{gathered}
\operatorname{Viab}_{F}(K):=\left\{x_{0} \mid \exists x(.), x(0)=x_{0} \text { and } \forall t=0, \ldots, T,\right. \\
x(t+1) \in F(x(t)), x(t) \in K\} .
\end{gathered}
$$

Trajectories visiting states outside the viability kernel are bound to fall below their sufficiency thresholds before the time horizon. From $C$-viable states, there exists at least one trajectory that remains above the threshold. To achieve this, a right decision in terms of harvest rates $h_{t}^{R}$ and $h_{t}^{r}$, whatever the perturbation $v_{t}^{P}$, must be taken at the right time in each $C$-viable state so as to remain in $K$.

The capture-viability kernel $\mathrm{Capt}_{F}(K, C, T)$ at time horizon $T$ of a target-set $C$ in $K$ under the dynamic $F$ is defined as the set of all states of $K$ from which there exists at least one solution that remains in $K$ until time $T$ and hitting the target $C \subset K$ at generation $T$ :

$$
\begin{aligned}
& \operatorname{Capt}_{F}(K, C, T):=\left\{x_{0} \mid \exists x(.), x(0)=x_{0} \text { and } \forall t\right. \\
& \quad=0, \ldots, T, x(t+1) \in F(x(t)), x(t) \in K, x(T) \in C\}
\end{aligned}
$$

Capture-viability kernels are computed by $C$-viability algorithms after the modification of the image $F(x)$ of $x$ into:

$\begin{cases}F(x) & \text { if } x(t) \notin C, \\ \operatorname{Clos}(\operatorname{Co}\{F(x)) \cup\{0\}\} & \text { if } x(t) \in C,\end{cases}$

where $\operatorname{Clos}(\operatorname{Co}(A))$ designates the closure of the smallest convex set containing the set $A$. We use Bonneuil (2006)'s $C$-viability algorithm, which is also presented in Bonneuil and Boucekkine (2016).

To obtain a viable solution which is also optimal in the sense of solving (1), we introduce the auxiliary variable $y$, and with it, the time variable $t$ which now appears as a state variable on its own, being involved in $y$ :

$$
\left\{\begin{aligned}
R_{t+1}= & R_{t}\left(K_{R r}-R_{t}-r_{t}\right)\left(1+v^{H}\right)\left(1-h_{t}^{R}\right) \\
& \times\left(\rho\left(1-c_{f}\right)\left(A_{t}+(1-s) a_{t}\right)+(1-\rho)\left(1-c_{f}+\epsilon\right)\right) \\
r_{t+1}= & r_{t}\left(K_{R r}-R_{t}-r_{t}\right)\left(1+v^{H}\right)\left(1-h_{t}^{R}\right) \\
& \times\left(\rho(1-s)\left(A_{t}+a_{t}\right)+1-\rho\right) \\
A_{t+1}= & A_{t} \rho\left((1-c) R_{t}+r_{t}\right)\left(1+v_{t}^{P}\right) \\
a_{t+1}= & a_{t} \rho(1-b)\left(1+v_{t}^{P}\right)\left(R_{t}+r_{t}\right) \\
t(\tau+1)= & t(\tau)+1 \\
y(t+1)= & y(t)-e^{-\xi t}\left(h_{t}^{R} R_{t}+h_{t}^{r} r_{t}\right)
\end{aligned}\right.
$$

with 
where (discrete) time is denoted by $\tau$ [then all state variables depend on $\tau$ through the state variable $t=t(\tau)=\tau$ ]. The problem has six dimensions and defines the augmented set-valued dynamic $F^{(a)}(x)$, with state constraints $K \times[0, T] \times \mathbb{R}^{+}$and target $C:=K \times\{T, 0\}$. The addition of the auxiliary variable $y$ participates in the elegant procedure leading to locate the viable maximum on the boundary in the direction of high $y$ of the capture-viability kernel associated with the augmented dynamic (15) (Bonneuil 2012b). Formally, this capture-viability kernel namely is

$\operatorname{Capt}_{F^{(\mathrm{a})}}\left(K \times[0, T] \times \mathbb{R}^{+}, K \times\{T, 0\}, T\right)$.

It comprises all maximum viable value functions $y(0)$. The $C$-viability algorithm of Bonneuil (2006), based on the stochastic minimization of the distance of trajectories starting from a given state to both the set of constraints and the target, is up to the task of dealing with six dimensions and yielding points on its boundary in direction of high $y$.

For each of the 1500 simulation runs, we drew the parameter values uniformly from intervals centered around the values given in Tellier and Brown (2007) and Moreno-Gàmez et al. (2013):

- the growth rate $v^{H} \in[0.03,0.07]$ of the host;

- the cost of fitness $c_{f} \in[0,0.15]$;

- the cost of inability to infect the RES allele $c \in[0.8,1.0]$;

- the cost $s \in[0.20,0.60]$ of being diseased;

- the cost of virulence $b \in[0.05,0.35]$;

- the cost $\epsilon \in[0.30,0.70]$ of activating and expressing the defense genes;

- the carrying capacity $K_{R r} \in[2.0,4.0]$;

- the probability $\rho \in[0.2,0.4]$ that the host encounters the parasite;

- the bounds $\nu_{\text {min }}^{P} \in[-0.05,-0.03]$ and $\nu_{\max }^{P} \in[0.03,0.05]$ of the growth rate of the parasite;

- the discount rate $\xi \in[0.01,0.03]$.

\section{Results}

Figure 1 shows a section of the four-dimensional $C$-viability kernel $\operatorname{Viab}_{F}(K)$ of the set $K$ defined by (5) under the dynamic $F$ defined in (2) and control constraints (3). It was built from 2500 viable points generated by Bonneuil (2006)'s $C$-viability algorithm.

From each state of this set, there exists at least one trajectory preserving genetic diversity [defined by (5)] until the time horizon $T$, but that is not necessarily economically optimal.

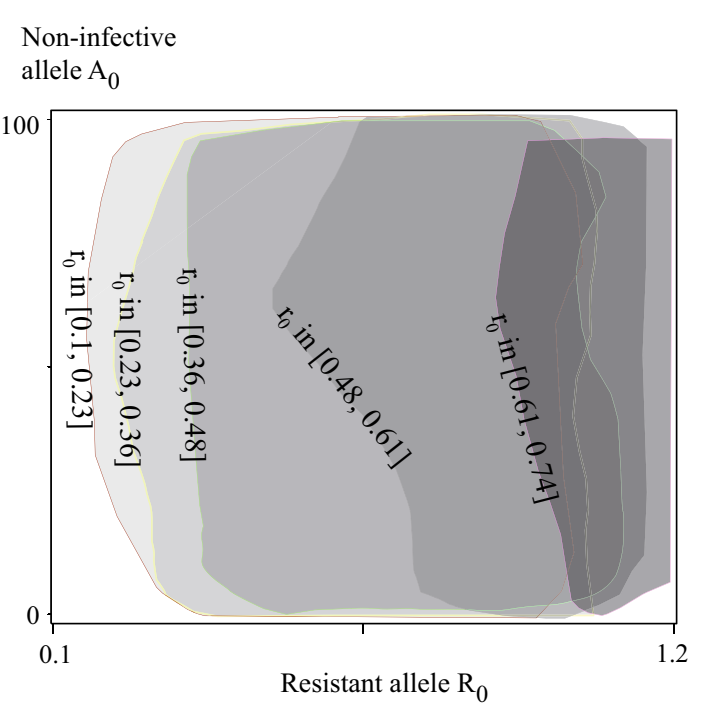

Fig. 1 Section of the $C$-viability kernel for infective allele $a_{0} \in[60,80]$ : from each state of the $C$-viability kernel, there exists at least one trajectory that remains in the set of constraints defined by (5) until the time horizon, here $\mathrm{T}=5$ generations, $p_{\min }=1 / 3$ and $p_{\max }=3$

\section{C-viability and C-viability-optimality}

Figure 1 shows that the viability kernel shrinks when $R_{0}$ and $r_{0}$ increase. This is due to the constraint $R / r \leq p_{\max }$. It also shows that the decrease accelerates for $r_{0}$ high and $A_{0}$ low, because an encounter with an infective parasite $a_{0}$ is more likely. The boundary of the $C$-viability kernel also curves when approaching low values of $A_{0}$ and $R_{0}$, because, then, $r_{0}$ prevails over $R_{0}$ and consequently, due to the prevailing of the infective allele $a_{0}$, most encounters are infective, rendering these states non-viable.

As we mentioned, Fig. 1 has no link with optimality yet. This requires the computation of $\operatorname{Capt}_{F^{(a)}}\left(K \times[0, T] \times \mathbb{R}^{+}, K \times\{T, 0\}, T\right)$, whose boundary in direction of high $y$, as we explained in the previous section, comprises the value functions $y(0)$ that are both viable and optimal. Figure 2 shows a section (at $A_{0}$ and $a_{0}$ restrained to a narrow range) of this boundary as a function of $R_{t}$.

When the resistant allele takes low and intermediate frequencies $R_{0}$, the carrying capacity is not attained and, as expected, the value function increases. Conversely, when both the resistant and the susceptible alleles are abundant, the value function decreases (curve associated with $\left.r_{0} \in[0.61,0.74]\right)$. All curves at $r_{0}$ fixed show a deceleration when the resistant allele increases. This corresponds to the decreasing return from having a less productive allele. The curves for $r_{0}$ low are limited on their right-hand side because of the constraint $R / r<p_{\max }$. 


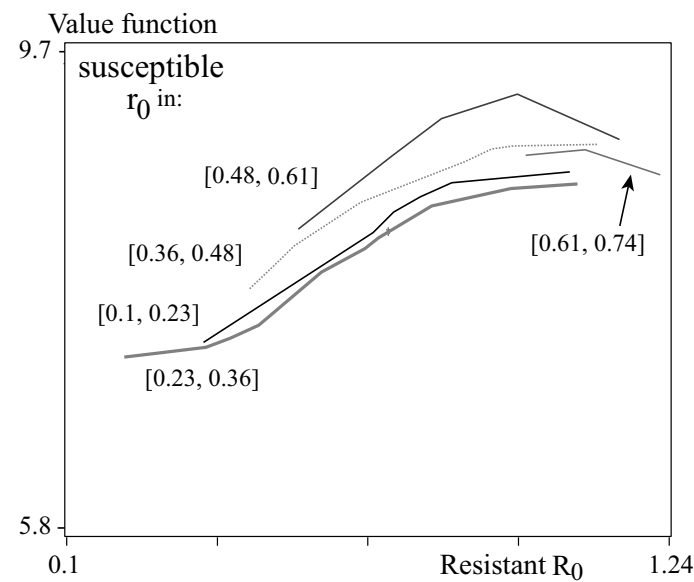

Fig. 2 Section of the capture-viability kernel for non-infective $A_{0} \in[60,80]$ and infective $a_{0} \in[80,100]$ showing the value function: from each state of the capture-viability kernel, there exists at least one trajectory that produces the maximal inter-temporal benefit and that remains in the set of constraints defined by (5) until the time horizon, here $\mathrm{T}=5$ generations

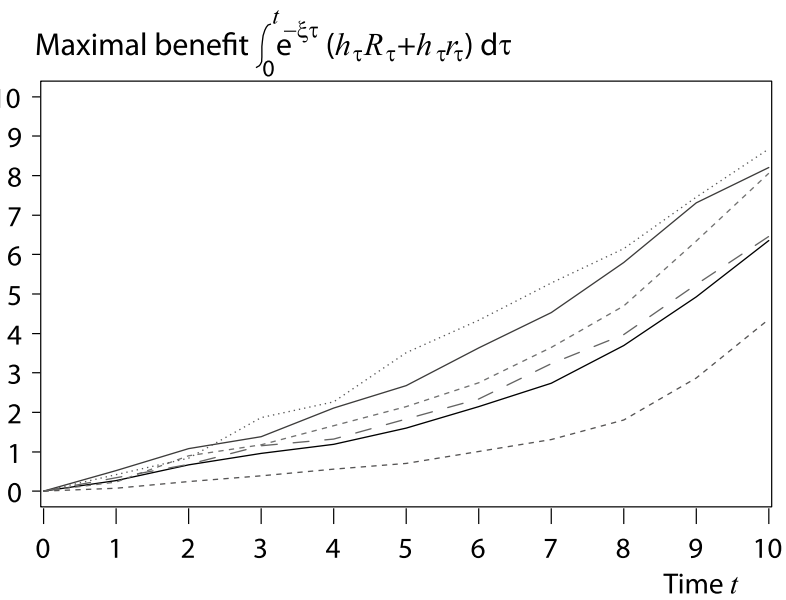

Fig. 3 Examples of optimal paths over time starting from the same initial state $\left(R_{0}, r_{0}, a_{0}, A_{0}\right)=(0.54,9.65,24.75,32.72), \mathrm{T}=10$ generations. From top to bottom at $t=7:\left(v^{H}, c_{f}, c, s, b, v_{\text {min }}^{P}, \epsilon, K_{R r}, \rho\right)=$ $(0.05,0.08,0.86,0.32,0.12,-0.03,0.06,3.79,0.36),(0.05,0.10,0.97$, $0.44,0.11,-0.02,0.05,3.65,0.23),(0.04,0.12,0.88,0.34,0.10,-0.02$, $0.05,3.73,0.37),(0.03,0.09,0.94,0.38,0.13,-0.03,0.06,3.36,0.38)$, $(0.05,0.11,0.84,0.45,0.13,-0.03,0.04,3.28,0.25),(0.06,0.11,0.86,0.42$, $0.08,-0.04,0.05,2.76,0.27))$

Figure 2 then represents the trade-off between the resistant and the susceptible alleles in a parasitized and constrained environment.

At a reviewer's request, Fig. 3 shows examples of trajectories of the inter-temporal benefit leading to the maximal value $y(0)$ [in fact, these trajectories were computed backward, in agreement to (15)]. The hierarchy of trajectories is not simple with respect to the parameters, because it depends on the linear combination of many parameters $\left(c_{f}, c, s, b, v^{P}, \epsilon, K_{R r}\right.$, and $\left.T\right)$ and initial conditions $\left(\rho R_{0} A_{0} / S_{0}, \rho R_{0} a_{0} / S_{0}, \rho r_{0} A_{0} / S_{0}, \rho r_{0} a_{0} / S_{0},(1-\rho) R_{0} / S_{0}\right.$, and $\left.S_{0}\right)$, where $S_{0}=\rho\left(R_{0}+r_{0}\right)\left(A_{0}+a_{0}\right)+(1-\rho)\left(R_{0}+r_{0}\right)$ is the population size of plants. That is why a regression of $y(0)$ on these parameters and initial conditions is necessary. Because of optimality, the values of the control variables along an optimal trajectory are entirely given by these initial conditions.

\section{Economic value as a function of initial states and parameters}

We compute 1500 points to describe the upper boundary of the capture-viability kernel in the direction of high $y$, for $p_{\min }=1 / 3$ and $p_{\max }=3$ as a typical case. In all the regressions below, the coefficient of $(1-\rho) r$ is set to 0 , because this variable is 1 minus the other proportions, so the coefficients of the other proportions are relative to the occurrence of the non-resistant plant not encountering the parasite, in proportion $(1-\rho) r$, taken as reference [taking another reference changes the coefficients, but not the differences (to be tested against 0 ) between two coefficients, as is usual].

The regression below (estimated with the SAS procedure "glm") describes the maximal value of the economic value function $y$ as a linear function of the initial values $R_{0}, r_{0}, a_{0}$, and $A_{0}$ of the $C$-viable states and the parameters, including the carrying capacity $K_{R r} \geq\left(R_{t}+r_{t}\right)$ and the mean values of the controls $\left(N=1500, \mathrm{R}^{2}=0.75\right)$ :

$$
\begin{aligned}
y_{0}= & -\underset{(0.08)}{0.68^{*}}+\underset{(0.06)}{0.08} \rho R_{0} A_{0} / S_{0}+\underset{(0.06)}{0.25^{*}} \rho R_{0} a_{0} / S_{0} \\
& +\underset{(0.08)}{0.20^{*}} \rho r_{0} A_{0} / S_{0} \\
& +\underset{(0.07)}{0.15^{*}} \rho r_{0} a_{0} / S_{0}+\underset{(0.11)}{0.28^{*}}(1-\rho) R_{0} / S_{0} \\
& +\underset{(\text { (ref.) }}{0}(1-\rho) r_{0} / S_{0} \\
& +\underset{(0.02)}{0.49^{*}} S_{0}+\underset{(0.02)}{0.04^{*}} c_{f}+\underset{(0.04)}{0.004 c} \\
& +\underset{(0.02)}{0.08^{*}} s+\underset{(0.02)}{0.004 b}+\underset{(0.02)}{0.002 v_{\min }^{P}} \\
& -\underset{(0.03)}{0.001} v_{\max }^{P}+\underset{(0.02)}{0.02 \epsilon}+\underset{(0.02)}{1.13^{*}} K_{R r}+\underset{(0.01)}{0.000} \xi \underset{(0.01)}{0.26 *} T,
\end{aligned}
$$

where the star denotes significance at the 5\% level, standard deviations in parentheses under the coefficients, $S=\rho(R A+R a+r A+r a)+(1-\rho)(R+r)$. The 
hierarchy of the coefficients reflects that of the influences of the determinants, because we have normalized them between 0 and 1, except the coefficients associated with the proportions of compounds of parasite and host $\rho R_{0} A_{0} / S_{0}, \rho R_{0} a_{0} / S_{0}, \rho r_{0} A_{0} / S_{0}, \rho r_{0} a_{0} / S_{0},(1-\rho) R_{0} / S_{0}$, and $(1-\rho) r_{0} / S_{0}$, because they sum to 1 . These proportions are thus collinear and one coefficient must be set to 0 ; here we take the coefficient of $(1-\rho) r_{0} / S_{0}$ arbitrarily (the interpretation does not change with the choice of the reference, because the effects are relative). The proportion of resistant hosts not encountering any parasite has a significantly lower effect on maximal benefit (coefficient $-0.28, \mathrm{SD}=0.11$ ) than the proportion of susceptible hosts not encountering any parasite (reference): resistance is a useless genetic burden in the absence of encounters.

Increasing the proportion of susceptible hosts encountering a parasite, be it infective or not $(0.15$ and 0.20 , SD $=0.07$ and 0.08 ), or increasing the proportion of resistant hosts encountering infective parasites $(0.25, \mathrm{SD}=0.06)$, increases the maximal viable benefit: encountering the parasite allows diversity in RES and res, whereas diversity is doomed to vanish in the absence of the parasite. These three proportions are not significantly different from each other. The proportion of resistant hosts encountering a non-infective parasite is not significantly different from the effects of these three proportions (coefficient $0.08, \mathrm{SD}=0.06$ ) consistently with the maintenance of diversity, but it is also not significantly different from the proportion of susceptible hosts not encountering any parasite (reference). This is consistent with the fact that these two cases imply no disease.

Apart from the effects of proportions of combinations of host and parasite, the hierarchy of importance of the different effects, because the parameters have been normalized in the regression, is reflected by the hierarchy of coefficients in absolute value: the carrying capacity $K_{R r}(1.13, \mathrm{SD}=0.02)$ has the major effect, followed by the population size $S_{0}$ $(0.49, \mathrm{SD}=0.02)$, the time horizon $(0.26, \mathrm{SD}=0.01)$, the cost $s$ of the disease $(-0.08, \mathrm{SD}=0.02)$, and the cost $c_{f}$ of fitness $(-0.04, \mathrm{SD}=0.02)$. The cost $\epsilon$ of activating and expressing the defense genes, the cost $c$ of inability to infect RES, the cost of virulence $b$, the bounds $v_{\min }^{P}$ and $v_{\max }^{P}$ of the growth rate of the parasite, and the discount rate $\xi$ have no significant effect, which come from their relatively low values.

Along the optimal paths leading to the maximal value $y_{0}$, the dependence of optimal harvest rates on state variables and other controls are estimated through "seemingly unrelated" regressions, which are in fact related by the correlation matrix of the residuals (Zellner 1962), with the argument time $t$ omitted on the right-hand side $\left[N=1500, \mathrm{R}^{2}=0.58\right.$ for $h^{R}(t), \mathrm{R}^{2}=0.43$ for $\left.h^{r}(t)\right]$ :

$$
\begin{aligned}
& h^{R}(t)=\underset{(0.03)}{0.42^{*}}-\underset{(0.03)}{0.14^{*}} v^{P}+\underset{(0.03)}{0.94^{*}} \rho R A / S+\underset{(0.02)}{0.70^{*}} \rho R a / S \\
& +\underset{(0.03)}{0.33^{*}} \rho r A / S+\underset{(0.03)}{0.22 *} \operatorname{\rho ra} / S+\underset{(0.02)}{0.29^{*}}(1-\rho) R / S \\
& +\underset{\text { (ref.) }}{0.00 *}(1-\rho) r / S+\underset{(0.02)}{0.47^{*}} S-\underset{(0.01)}{0.08 *} c_{f}-\underset{(0.02)}{0.02 c} c \\
& -\underset{(0.02)}{0.02 s}+\underset{(0.01)}{0.01 b}-\underset{(0.01)}{0.03^{*}} \epsilon+\underset{(0.01)}{0.71^{*}} K_{R r}+\underset{(0.01)}{0.00 \xi}-\underset{(0.01)}{0.07 *} T \text {, }
\end{aligned}
$$

for the harvest rate $h^{R}$ of the allele "RES", and

$$
\begin{aligned}
h^{r}(t)= & \underset{(0.03)}{0.18^{*}}-\underset{(0.03)}{0.12^{*}} \nu^{P}-\underset{(0.03)}{0.30^{*}} \rho R A / S-\underset{(0.02)}{0.36^{*}} \rho R a / S \\
& +\underset{(0.03)}{0.15^{*}} \rho r A / S+\underset{(0.03)}{0.27^{*}} \rho r a / S-\underset{(0.02)}{0.75^{*}}(1-\rho) R / S \\
& +\underset{(\text { (ref. }))}{0.00}(1-\rho) r / S+\underset{(0.03)}{0.42^{*}} S+\underset{(0.02)}{0.02 c_{f}}-\underset{(0.02)}{0.03 c} \\
& -\underset{(0.01)}{0.06 *} s+\underset{(0.01)}{0.003 b}-\underset{(0.01)}{0.00 \epsilon}+\underset{(0.01)}{0.63^{*}} K_{R r} \\
& +\underset{(0.01)}{0.00 \xi}-\underset{(0.01)}{0.04^{*}} T,
\end{aligned}
$$

for the harvest rate $h^{r}$ of the allele "res". The correlation between the two regressions is 0.50 , which indicates that the two harvest rates vary mostly in the same direction, which is consistent with the logic of producing a value function while maintaining $R / r$ within the constraints.

As expected, the carrying capacity $K_{R r}$ again has one of the most important effects ( 0.71 and $0.63, \mathrm{SD}=0.01$ and 0.01 ) on both harvest rates (the more the plant population can grow, the better). The more abundant the resistant allele, the higher its harvest rate $[0.94, \mathrm{SD}=0.03$, for $\rho R A / S ; 0.70, \mathrm{SD}=0.02$, for $R a / S$; and $0.29, \mathrm{SD}=0.02$, for $(1-\rho) R / S$ - the order of the coefficients reflects the trade-off between benefit maximization and genetic diversity]. To preserve genetic diversity, the more the susceptible allele res is present, the less the resistant allele RES is harvested $[-0.75, \mathrm{SD}=0.02$, for $(1-\rho) R / S ;-0.36$ $\mathrm{SD}=0.02$ for $\rho R a / S ;-0.30, \mathrm{SD}=0.03$, for $\rho R A / S]$. Symmetrically, the more abundant the susceptible plant, the more it is harvested $(0.27, \mathrm{SD}=0.03$, for $\rho \mathrm{ra} / \mathrm{S}$ and $0.15, \mathrm{SD}=0.03$, for $A / S$, so almost twice as likely in the presence of the infective allele as in the presence of the non-infective allele). This helps to eliminate the parasite and give an additional chance to the remaining susceptible alleles. Again, in order to maintain genetic diversity, the more the resistant allele is present, the less the susceptible allele is harvested $(0.33, \mathrm{SD}=0.03$, for $\rho r A / S$ and 0.22 , $\mathrm{SD}=0.03$, for $\rho r a / S$ ).

The total size $S$ of the plant population favors both harvest rates $(0.47, \mathrm{SD}=0.02$, for RES and $0.42, \mathrm{SD}=0.03$, for 


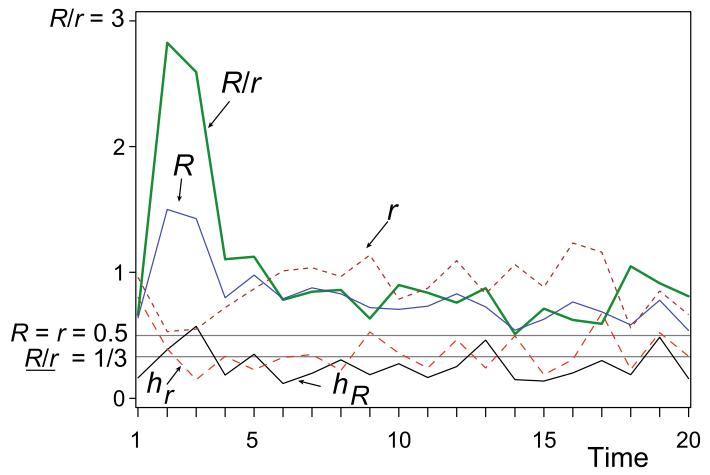

Fig. 4 Example of a simulated trajectory remaining within the constraints and approaching them. $\mathrm{T}=20$ generations

res). The higher the growth rate $v^{P}$ of the parasite, the lower the harvest rates $(-0.14, \mathrm{SD}=0.03$, for RES and -0.12 , $\mathrm{SD}=0.03$, for res). The higher the $\cos t c_{f}$ of fitness to resistant hosts that do not encounter a pathogen, the less these hosts should be harvested $(-0.08, \mathrm{SD}=0.01)$, but consistently, there is no significant effect on the harvesting of susceptible alleles $(0.02, \mathrm{SD}=0.02)$. The longer the time horizon, the more plants should be spared $(-0.07, \mathrm{SD}=0.01$, for RES and $-0.04, \mathrm{SD}=0.01$, for res). The cost $s$ of the disease penalizes the susceptible allele $(-0.06, \mathrm{SD}=0.01)$, but consistently, not the resistant allele. Symmetrically, the cost $\epsilon$ of activating and expressing defense genes penalizes the resistant allele $(-0.03, \mathrm{SD}=0.01)$ but not the susceptible allele. The costs $c$ of inability to infect the virus and the virulence $b$ play no significant role in harvest rates.

Along the optimal viable path, the resistant strain "RES" has the strongest effect $(0.94, \mathrm{SD}=0.03)$ and the susceptible strain "res" must be spared, because it is threatened. That is why both harvest rates increase on the optimal path when "res" is more frequent (coefficients $0.33,0.21,0.14$, and 0.26). When "RES" increases in frequency, whatever the combination with the parasite (coefficients $0.93,0.69$, and 0.28 ), its harvest rate increases, but, in order to preserve $R / r$, the more fragile "res" must be spared, which implies that $h^{r}$ must decrease (coefficients $-0.29,-0.36$, and -0.74 ). As expected, coefficients of the population size $S$ of plants and of the carrying capacity $K_{R r}$ are positive and significant. The longer the period of exploitation $T$, the more the plant must be spared (coefficients -0.004 and -0.003 ). A more rapid population growth of the parasite leads to increased harvesting of both alleles (coefficients 0.24 and 0.17 ).

Figure 4 shows the example of an optimal trajectory remaining in the set of constraints $K$. The $C$-viability condition demands that at each time, the direction of the system belongs to the contingent cone of $K$. Figure 4 shows that the trajectory approaches the condition $R / r \geq p_{\max }$ at $t=2$ as well as the condition $r \geq r_{\text {min }}=0.5$ : then, as the presented trajectory is a solution to the viable optimum, it remains in $K$ and $h_{R}$ and $h_{r}$ are selected under varying $v^{P}$ such that the trajectory does not cross the boundary of $K$, here $R / r=p_{\max }$ together with $r=r_{\min }$. At $t=2, h_{r}$ decreases and $h_{R}$ increases, allowing $r$ to stop decreasing and $R / r$ to stop increasing. Likewise, at $t=14$, the harvest rate $h_{R}$ decreases and $h_{r}$ increases, allowing the trajectory to remain over the constraints $R / r=p_{\min }$ and $R=R_{\min }=0.5$. This Fig. 4 shows therefore a case where $C$-viability conditions (that the velocity of the system must belong to the contingent cone of $K$ ) interfere with optimality.

\section{Genetic distance and value function}

Brock and Xepapadeas (2003, p. 1597) quantify genetic diversity by means of the Shannon distance, here equal to

$d_{1}=-R /(R+r) \ln (R /(R+r))-r /(R+r) \ln (r /(R+r))$,

and the Simpson distance, here equal to

$d_{2}=1-(R /(R+r))^{2}-(r /(R+r))^{2}=2 r R(r+R)^{-2}$.

These distances are null at $R=0$ or $r=0$, achieve their maximal values at $r=R$, with $d_{1}=\ln (2)$ and $d_{2}=0.5$, and their minimum values on $K$ at $d_{1} \approx 0.56$ and $d_{2}=0.375$ for both $R / r=p_{\text {min }}=1 / 3$ and $R / r=p_{\max }=3$.

In (17), we saw that $R_{0}$ and $r_{0}$ play different roles, depending on the presence of the infective parasite. This asymmetry makes the Shannon or the Simpson distances ambivalent for characterizing the value function: a single value of a distance corresponds to two half-lines in $(R, r)$, then to high and low values of $R_{0}$ or $r_{0}$. We regress the log-value function $y_{0}$ on the log-distance $\ln \left(d_{1}\right)$ and the Shannon distance $d_{1}$ to capture possible nonlinearity (all covariates normalized between 0 and 1 to make coefficients comparable) $\left(N=1500, \mathrm{R}^{2}=0.60\right)$ :

$$
\begin{aligned}
& \ln \left(y_{0}\right)=-\underset{(33.92)}{116.95^{*}}-\underset{(23.70)}{80.66^{*}} \ln \left(d_{1, \max )}+\underset{(36.41)}{123.33^{*} d_{1, \max }}\right. \\
& -\underset{(0.06)}{0.01 \ln }\left(d_{1, \max }\right) \rho A_{0}
\end{aligned}
$$

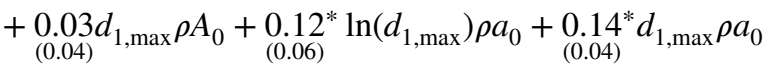

$$
\begin{aligned}
& -\underset{(0.55)}{0.06 c_{f}}+\underset{(0.17)}{0.11 c} c-\underset{(0.14)}{0.43^{*}} s-\underset{(0.55)}{0.22 b}-\underset{(1.65)}{1.59} v_{\min }^{P}-\underset{(1.05)}{1.14} v_{\max }^{P} \\
& -\underset{(1.12)}{1.22 \epsilon}+\underset{(0.03)}{0.89^{*}} K_{R r}+\underset{(0.01)}{0.01 \xi}+\underset{(0.002)}{0.051^{*} T}
\end{aligned}
$$

( $N=1500, R^{2}=0.60$, “*” again denotes significance at the $5 \%$ level, standard deviations in parentheses under the coefficients), where $X_{\max }$ denotes the mean of the variable $X$ over the trajectory. In addition to the expected significant positive effects of the carrying capacity $K_{R r}$ and the time horizon $T$, Eq. (22) shows that $y_{0}$ is a convex function of distance, controlling for other covariates. The maximal value 
over the Shannon distance of this maximal benefit $y_{0}$ does not depend significantly on the frequency $\rho A_{0}$ of encounters with the non-infective parasite $(-0.01, \mathrm{SD}=0.06$, and 0.03 , $\mathrm{SD}=0.04$ ), but does depend on the frequency $\rho a_{0}$ of encounters with its infective allele (coefficients $0.12, \mathrm{SD}=0.06$, and $0.14, \mathrm{SD}=0.04)$ : when $\rho a_{0}$ is low, the maximal benefit is higher for $d_{1}=1 / 3$ or 3 , that is, on the boundary of the constraints, with the risk of leaving the set of constraints and consequently fail to comply with genetic diversity; if $\rho a_{0}$ is high, then the maximal benefit is higher for $d_{1}=\ln (2)$, which corresponds to the resistant and the susceptible alleles in the same proportions. This has the advantage of being far from the constraints defining diversity, but $\rho a_{0}$ must be high enough. Thus, effective management consists, if the prevalence values of the plant alleles can be adjusted, of starting from the initial conditions in RES and res in the proportions $1 / 3$ or 3 if $\rho a_{0}$ is low or in equal proportions if $\rho a_{0}$ is high. This recommendation holds only in order to maximize the maximum benefit over $\rho a_{0}$, when the manager makes decisions based on genetic distance, which, as we mentioned, is ambivalent, in contrast to Eq. (17), which thus should be preferred for management.

\section{Conclusion}

The $C$-viability framework solves the question of measuring the economic value of genetic diversity out of equilibrium and with unpredictable prevalence of the parasite. We treated the host-parasite case, each having two alleles, to show that combining economic rationality with genetic diversity reveals that the presence of the infective allele of the parasite is necessary, and that harvest rates must be varied according to the growth rate of the parasite, the encounter rate, and the time horizon. We showed that, when the infective parasite is abundant, genetic diversity allows greater economic benefit.

Here we have adapted economic calculus to genetics in its full heterogeneity. In order to remain diverse enough, that is, $C$-viable with respect to the fixed range of variation of the ratio $R / r$, against any variation of the parasite growth rate, how should farmers harvest? The answer is: remain on the upper boundary of the capture-viability kernel of $K \times[0, T] \times \mathbb{R}^{+}$with target $K \times\{T, 0\}$ defined in (9) under the dynamics (15). Then, the value function is optimal and genetic diversity is allowed by the maintenance of allele frequencies within sufficiency constraints. The $C$-viable and optimal rates $h^{R}$ and $h^{r}$ constitute the regulation law, which enables the system to perpetuate itself while preserving genetic diversity and yielding the maximal value function.

\section{References}

Aubin J-P (1991) Viability theory. Springer, Boston

Barrett JA (1980) Pathogen evolution in multilines and variety mixtures. Z Pflanzenk Pflanzens 87:383-396

Beissinger SR, McCullough DR (2002) Population viability analysis. University of Chicago Press, Chicago

Bonneuil N (1994a) Malthus, Boserup and population viability. Math Popul Stud 4(5):107-119

Bonneuil N (1994b) Capital accumulation, inertia of consumption and norms of reproduction. J Popul Econ 7:49-62

Bonneuil N (1998) Population paths implied by the mean number of pairwise nucleotide differences among mitochondrial DNA sequences. Ann Hum Genet 62:61-73

Bonneuil N (2003) Making ecosystem models viable. Bull Math Biol 65:1081-1094

Bonneuil N (2006) Computing the viability kernel in large state dimension. J Math Anal Appl 323(2):1444-1454

Bonneuil N (2010) Diversity of preferences in an unpredictable environment. J Math Econ 46:965-976

Bonneuil N (2012a) Multiallelic polymorphism maintained by unpredictable migration and selection. J Theor Biol 293:189-196

Bonneuil N (2012b) Maximum under continuous-discrete-time dynamic with target and viability constraints. Optimization 61(8):901-913

Bonneuil N, Boucekkine R (2014) Viable Ramsey economies. Can J Econ 47(2):421-444

Bonneuil N, Boucekkine R (2016) Optimal transition to renewable energy with threshold of irreversible pollution. Eur J Oper Res 248:257-262

Bonneuil N, Saint-Pierre P (2000) Protected polymorphism in the two-locus haploid model with unpredictable fitnesses. J Math Biol 40(3):251-277

Bonneuil N, Saint-Pierre P (2002) Minimal number of generations out of polymorphism in the one-locus two-allele model with unpredictable fertilities. J Math Biol 44(6):503-522

Brock WA, Xepapadeas A (2003) Valuing biodiversity from an economic perspective, a unified economic, ecological, and genetic approach. Am Econ Rev 93(5):1597-1614

Burdon JJ, Thrall PH (2003) The fitness costs to plants of resistance to pathogens. Genome Biol 4:227

Edwards PJ, Abivardi C (1998) The value of biodiversity: where ecology and economy blend. Biol Conserv 83(3):239-246

Gu YQ, Wildermuth MC, Chakravarthy S, Loh Y-T, Yang C, He X, Han Y, Martin GB (2002) Tomato transcription factors Pti4, Pti5, and Pti6 activate defense responses when expressed in Arabidopsis. Plant Cell 14:817-831

Hastings A (1988) Food web theory and stability. Ecology 69:1665-1668

Heal G (2000) Nature and the marketplace: capturing the value of ecosystem services. Island Press, Washington, DC

Henderson DR (2008) Present value. Concise encyclopaedia of economics, 2nd edn. Library of Economics and Liberty, Indianapolis

Law R, Morton D (1996) Permanence and the assembly of ecological communities. Ecology 77(3):762-775

Leonard KJ (1977) Selection pressures and plant pathogens. Ann NY Acad Sci USA 287:207-222

Meinarda Y, Dereniowskad M, Gharbi J-S (2016) The ethical stakes in monetary valuation methods for conservation purposes. Biol Conserv 199:67-74

Moreno-Gàmez S, Stephan W, Tellier A (2013) Effect of disease prevalence and spatial heterogeneity on polymorphism maintenance in host-parasite interactions. Plant Pathol 62(Suppl. 1):133-141 
Morin PJ, Lawler SP (1995) Food web architecture and population dynamics: theory and empirical evidence. Annu Rev Ecol Syst 26:505-529

Morris WF, Doak DF (2002) Quantitative conservation biology. Sinauer Associates, Inc., Sunderland

Nagumo M (1942) Über die Lage der integralkurven gewöhnlicher Differentialgleichungen. Proc Phys Math Soc Jpn 24:551-559

Nijkamp P, Vindigni G, Nunes P (2008) Economic valuation of biodiversity: a comparative study. Ecol Econ 67:217-231

Nuismer SL, Otto SP (2005) Host-parasite interactions and the evolution of gene expression. PLoS Biol 3(7):e203

Perron O (1915) Ein Neuer Existenzbeweis für die Integrale der Differentialgleichung $y^{\prime}=f(x, y)$. Math Ann 76:471-484

Pianka ER (1978) Evolutionary ecology. Harper and Row Publishers, New York

Slatkin M, Hudson RR (1991) Pairwise comparisons of mitochondrial DNA sequences in stable and exponentially growing populations. Genetics 129:555-562

Solow A, Polasky S, Broadus J (1993) On the measurement of biological diversity. J Environ Econ Manag 24(1):60-68

Spash CL (2015) Bulldozing biodiversity: the economics of offsets and trading-in. Nat Biol Conserv 192:541-551

Tellier A, Brown JKM (2007) Stability of genetic polymorphism in host-parasite interactions. Proc R Soc Lond B 274:809-817
Tellier A, Brown JKM (2011) Spatial heterogeneity, frequency-dependent selection and polymorphism in host-parasite interactions. BMC Evol Biol 11:319

Tellier A, Moreno-Gámez S, Stephan W (2014) Speed of adaptation and genomic footprints of host-parasite coevolution underarms race and trench warfare dynamics. Evolution 68(8):2211-2224

Ten Kate MM, Laird SA (2004) The commercial use of biodiversity. Earthscan, London

Weitzman ML (1992) On diversity. Q J Econ 107(2):363-406

Weitzman ML (1993) What to preserve? An application of diversity. Theory to crane conservation. Q J Econ 108(1):157-183

Weitzman ML (1998) The Noah's Ark problem. Econometrica 66(6):1279-1298

Wengera SJ, Leasurea DR, Dauwalterb DC et al (2017) Viability analysis for multiple populations. Biol Conserv 216:69-77

Williamson M (1987) Are communities ever stable? In: Gray AJ, Crawley MJ, Edwards PJ (eds) Colonization, succession and stability. Blackwell Scientific, Oxford

Zellner A (1962) An efficient method of estimating seemingly unrelated regression equations and tests for aggregation bias. J Am Stat Assoc 57:348-368 